\title{
Evaluation of Game Engines for Simulated Surgical Training
}

\author{
Stefan Marks \\ Division for Biomedical Imaging and Visualization \\ Department of Computer Science \\ University of Auckland, New Zealand \\ Email: dev.stefan.marks@gmx.net
}

\author{
John Windsor \\ Advanced Clinical Skills Center \\ Department of Surgery \\ Faculty of Medical and Health Sciences \\ University of Auckland, New Zealand \\ Email: j.windsoreauckland.ac.nz
}

\author{
Burkhard Wünsche \\ Division for Biomedical Imaging and Visualization \\ Department of Computer Science \\ University of Auckland, New Zealand \\ Email: burkhardecs.auckland.ac.nz
}
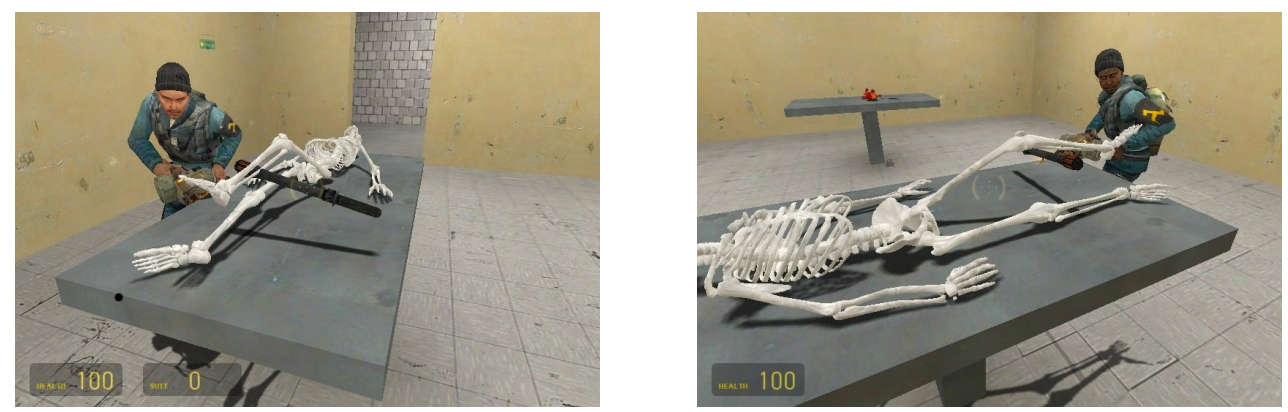

Figure 1: Screenshots of an interactive simulation scenario implemented with the Half-Life 2 engine. One user (left figure) is moving the skeleton's leg with a stick.

\begin{abstract}
The increasing complexity and costs of surgical training and the constant development of new surgical procedures has made virtual surgical training an essential tool in medical education. Unfortunately, commercial tools are very expensive and have a small support base. Game engines offer unique advantages for the creation of highly interactive and collaborative environments.
\end{abstract}

This paper examines the suitability of currently available game engines for developing applications for medical education and simulated surgical training. We formally evaluate a list of available game engines for stability, availability, the possibility of custom content creation and the interaction of multiple users via a network. Based on these criteria, three of the highest ranked engines are used for further case studies.

We found that in general it is possible to easily create scenarios with custom medical models that can be cooperatively viewed and interacted with. Limitations in physical simulation capabilities make some engines unsuitable for fully interactive applications, but they can be used in combination with predefined animations. We show that overall game engines represent a good foundation for low cost virtual surgery applications and we discuss technologies which can be used to further extend their physical simulation capabilities.

CR Categories: I.3.5 [Computer Graphics]: Computational Geometry and Object Modeling-Physically based modelling; I.6.8 [Simulation and Modeling]: Types of Simulation-Gaming; J.3 [Life and Medical Sciences]: Medical information systems; K.3.1 [Computers and Education]: Computer Uses in EducationCollaborative learning

Copyright $@ 2007$ by the Association for Computing Machinery, Inc.

Permission to make digital or hard copies of part or all of this work for personal or classroom use is granted without fee provided that copies are not made or distributed for commercial advantage and that copies bear this notice and the full citation on the first page. Copyrights for components of this work owned by others than ACM must be honored. Abstracting with credit is permitted. To copy otherwise, to republish, to post on servers, or to redistribute to lists, requires prior specific permission and/or a fee. Request permissions from Permissions Dept, ACM Inc., fax +1 (212) 869-0481 or e-mail permissions@acm.org.

GRAPHITE 2007, Perth, Westem Australia, December 1-4, 2007.

(C) 2007 ACM 978-1-59593-912-8/07/0012 $\$ 5.00$
Keywords: game engines, physically-based animation, collaborative environments, simulation systems

\section{Introduction}

The rising complexity and costs of surgical training and the development of new surgical procedures make virtual surgery simulations increasingly important. Bradley [2006] lists the three major steps in the development of surgical simulators: In 1960, Åsmund Lærdal developed the prototype mannequin. The first computer controlled anaesthetics simulator and the animated mannequin SimOne were introduced 1969. Now, in 2007, high fidelity simulators are capable of satisfying the optical and physical aspect of a surgical simulation as well as educational concepts of the medical curriculum.

Most commercially available simulators cover the area of endoscopic respectively laparoscopic procedures (e.g. Procedius MIST [Mentice 2007], LapSim [Surgical Science 2007], LAP Mentor [Simbionix 2007], VEST System One [select IT VEST Systems AG 2007], LapVR [Immersion Corporation 2007], SEP [SimSurgery 2007], EndoTower [Verefi Technologies 2007], HystSim [Westwood et al. 2005]). This kind of procedure requires well developed skills of the surgeon with respect to coordination of the camera and the surgical instruments that are not in direct view and are represented only on a 2D screen, sometimes with changed orientation due to camera rotation. The above mentioned systems are all able to train basic procedures in camera and instrument handling, before training the medical and surgical aspects.

Nevertheless, those technical skills are not the only necessity for a surgeon. The AGCME Outcome project [ACGME 1999] lists six general competencies which include other skills like patient care, medical knowledge, the ability to continuously learn and improve by practise, interpersonal and communication skills, professionalism, and the awareness of the health care system with its resources and demands as a whole.

Most of the mentioned simulator systems only train the technical and procedural skills of a surgical procedure, but lack the other 
aspects of the above list. Few physical simulators with mannequins (e.g. MedSim-Eagle [CISL 2007]) enable small groups of residents to practise the cooperative aspects (i.e. interpersonal and communication skills) of medical or surgical procedures, but are very costintensive and thus likely to be unaffordable for most institutions.

One factor that is responsible for high costs of surgical simulators is the fact that certain parts of them are repeatedly reinvented. All simulators need at least graphical output capable of displaying 3-dimensional models with a high level of realism and user interfaces for operating and configuration of the simulator, an underlying physical simulation model, and event handling for input devices (see figure 2). Some simulators are capable of adding the audible aspect of a surgical procedure and thus need a module for sound generation.

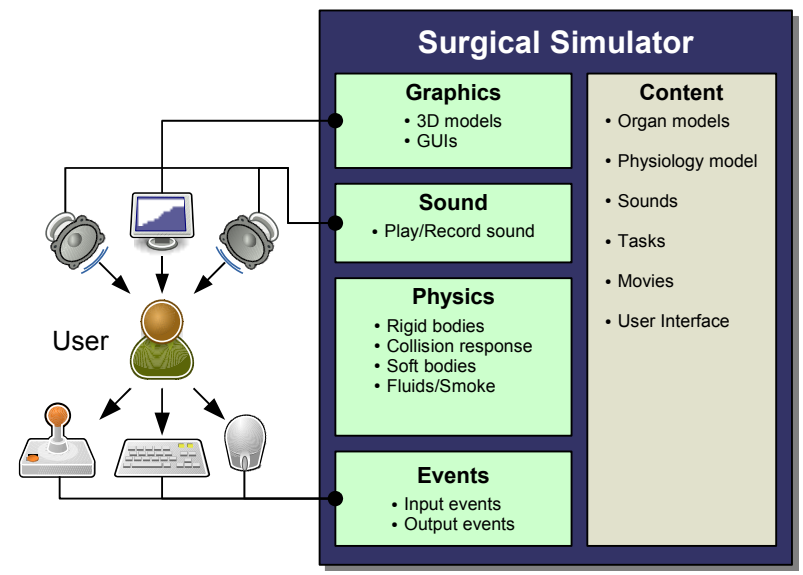

Figure 2: Functional blocks of a surgical simulator.

There have been attempts to create extensible frameworks for building surgical simulators upon (e.g. SPRING [Montgomery et al. 2002], GiPSi [Çavuşoğlu et al. 2006], SOFA [Allard et al. 2007], ESQUi [Rodriguez-Florido et al. 2006]). They all incorporate the above mentioned modules and a variety of mathematical models for the physical simulation and interaction. But except for SPRING (ironically the oldest project in the list) they all lack the capability of networking with other simulators to build collaborative scenarios. Even worse, sound support is not built into one of them.

This paper examines the possibility to build surgical simulators upon a software system that incorporates all of the above mentioned features and is in addition highly developed, well tested and supported and available at low costs: Game Engines.

\section{Game Engines}

The use of games or game engines for medical education is a little explored research subject with many aspects still to be investigated. One reason for this might be the incoherency of the seriousness of medicine and the playful, sometimes violent character of computer games. Nevertheless, game engines offer a vast pool of useful concepts and resources in technical as well as in educational aspects.

Projects like the "Serious Game Initiative" [Serious Games Initiative 2007b] focus on offering help to "organize and accelerate the adoption of computer games for a variety of challenges facing the world today." A subproject founded by this initiative is "Games for Health" [Serious Games Initiative 2007a], focusing mainly on games used in various health care sectors.
Previous authors have so far concentrated on applications where the game content was about learning facts, rather than tasks and procedures. For example, Wünsche et al. [2005] have examined how game engines can be used for visualising medical datasets, and Mackenzie et al. [2003] utilise a game engine for anatomical education. However, so far nobody has focused on the cooperative aspect in the simulation of complex medical procedures.

\subsection{Game Engine Design}

A game engine is a complex software system necessary for developing and playing games. Two different games with the same underlying engine differ by the game content, i.e. graphics, sounds, storyline. Game engines build a bridge between this content and the underlying hardware. With the help of an operating system abstraction layer, the same game content can be run on many platforms (e.g. Windows, Linux, XBox) without change.

Modern game engines consist of all or a subset of functional blocks depicted in figure 3 .

The Graphics Engine loads, displays, manipulates and manages all the data related to graphical content and visual effects. 3D models of objects, landscapes, buildings, objects, animals, and players can be loaded, textured, lit, and animated. Additional effects (e.g. blurring, lens distortion, depth of field) can be added to enhance the visual realism. Particle systems are utilised to simulate fire, smoke, bubbles, blood, etc.

All audible content like sound effects, ambient noise, and music is handled by the Audio Engine. In connection with modern soundcards it is possible to simulate acoustic obstruction by objects, environments other than air, reverb, Doppler effect and the spatial position of sound sources.

The total memory usage of game content is often higher than the memory provided by the gaming platform. Because not all of this data is needed simultaneously, the Memory Management is responsible for purging unused content from memory and in turn providing and managing requested memory for new content. Modern engines parallelise tasks like graphics, sound, physics, and AI. To balance the workload of all these tasks efficiently, especially on multiprocessor platforms, the Process Management is utilised.

Another essential part is the Event handling. Input devices, like joysticks, mice, keyboards, and gamepads generate events as well as network, timers, other components of the gaming hardware, game scripts and many other sources. These events are handled, filtered and distributed by one central event loop.

Some media like music or video does not need to be loaded into memory before being played back, but can instead be streamed to save precious memory resources. The Streaming mechanism is also capable of loading resources via the network from other servers.

Realistic behaviour of game objects has become more and more important in the recent years. The Physics Engine implements advanced mathematical models for calculating rigid body simulations of arbitrarily shaped and articulated objects (e.g. vehicles, machines). With the development of highly sophisticated physics engines like Havok Physics ${ }^{\mathrm{TM}}$ [Havok 2007] or PhysX ${ }^{\mathrm{TM}}$ [Ageia Technologies 2007], the simulation of soft bodies, cloth, fluids, and smoke has become possible, accelerated either by specialised hardware or the computational power of the graphics hardware [Geer 2006].

Artificial intelligence, provided by the AI Engine, is needed for controlling Non-Player Characters (NPC), the computer controlled antagonists in games. NPCs have to make decisions about how to 


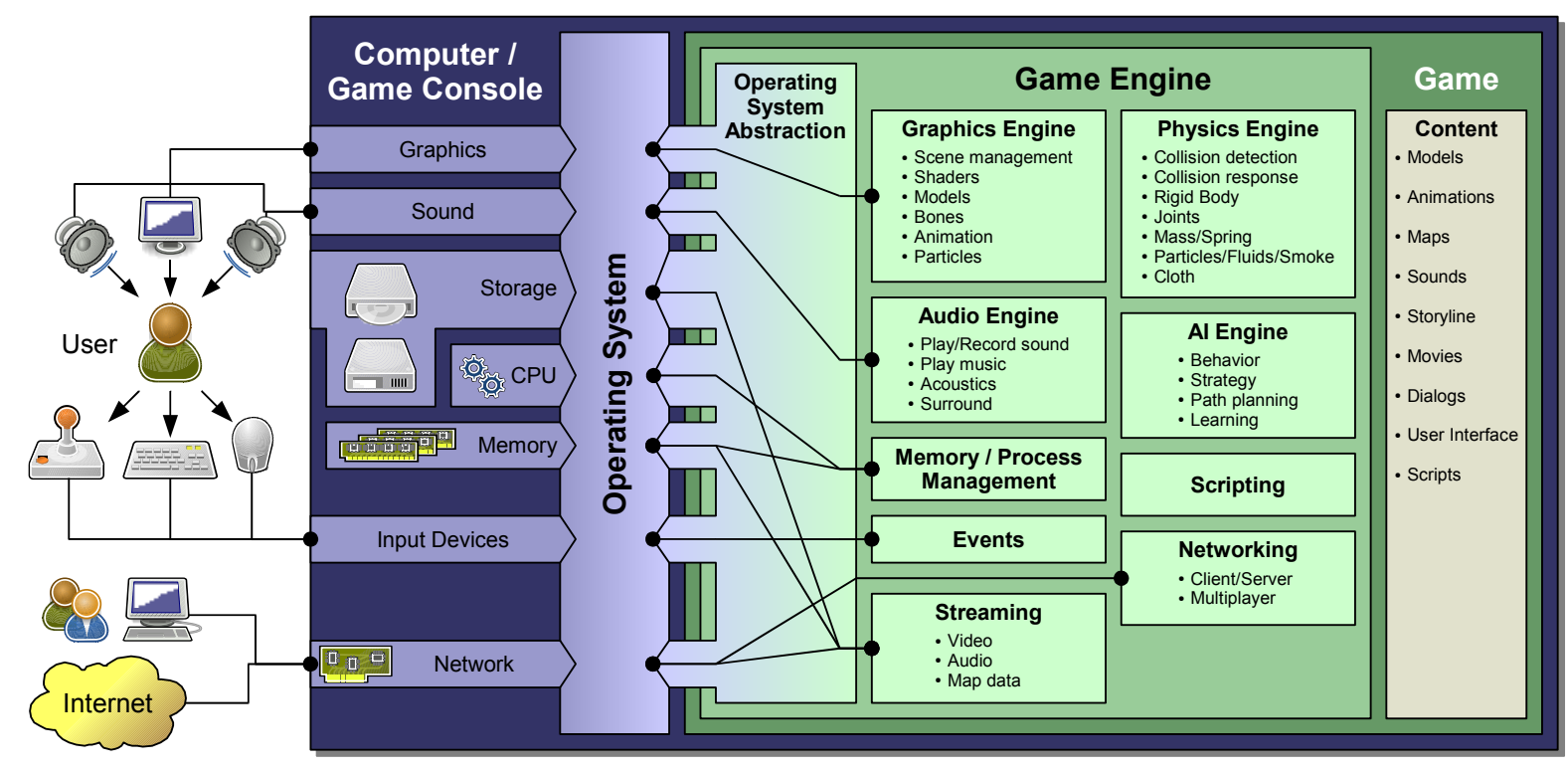

Figure 3: Functional Blocks of a Game Engine.

follow, avoid or ambush the player, and how to react to aggressive or defensive actions in a realistic and effective manner. AI Engines incorporate computer science topics like neural networks, state machines, $\mathrm{A}^{*}$ search, and much more.

The flexibility of game engines is their greatest strength in creating manifold content. This is achieved by Scripting languages that allow an immediate access to the functions of the game engine. By scripts, the game content gets its typical "fingerprint", how a game is to be played and controlled, how the story develops, and how interactive and immersive the game environment appears.

The final important functional block of a game engine is Networking. By sending the state, movement and other information of each player and NPC over the network, other connected human players can collaborate in a game, because they all see the identical state of the game world at the same moment. The networking functions cope with network problems like packet loss or different runtimes of data packets from clients to servers and vice versa.

\subsection{Technical Advantages}

Since the game market is incredible competitive and incorporates both large established and innovative new vendors, game engines are constantly updated and utilise the latest graphics hardware and graphics algorithms. In addition, since most users are unable to constantly update their machines, game engines are designed for handling the same game content on hardware with different speed, memory size and features.

Playing computer games is no longer an action for individuals but has evolved into multiplayer gaming, bringing together several thousand players at the same time [Chen et al. 2006]. Consequently, many game engines incorporate properly constructed and tested network support that serves well in connecting multiple users for cooperatively accomplishing tasks, even worldwide, unrestricted by location and physical boundaries. Built-in support for recording and playing sound over the network enables the players to communicate in a natural way to coordinate their actions. Textual input of messages serves as an alternative way.
Due to the fact that games are marketed internationally, game engines are able to deal with different languages. User interfaces, sound support and input devices can be customised accordingly.

After games have been introduced to the market, they are constantly and intensively used by customers which results in massive feedback about errors and flaws. After some months and sometimes only weeks, patches are available to fix these issues. During this time, a large number of developers will have built up, who have gathered experience in modifying the game content. They form an international support community, often willing to help others when problems arise in building custom content for a game engine.

\subsection{Important Features}

One major important aspect for medical simulation is visual realism. With game engines supporting the most modern graphics hardware, this issue can easily be addressed. To enhance the realism in their hystheroscopy simulator, Bachofen et al. introduces bump mapping, spotlights, shadows, lens distortion, depth of field, bubbles, and floating tissue [Bachofen et al. 2005]. This list is only a subset of effects used in modern games, as the screenshots in figure 4 illustrate.

The acoustic environment of a medical procedure is also an important part of a simulation [Westwood et al. 2005] and can also be easily and accurately simulated with the features available in game engines. It may provide audible feedback of instruments used during medical procedures as well as reactions of the simulated patient, like pain or relief.

The physical simulation of objects in games is a relatively young area and thus does not yet cover the mathematically demanding aspects of soft tissue simulation (e.g. [Delingette 1998; Cotin et al. 1999; Lim and De 2007]) or cutting (e.g. [Dworzak and Gu 2007]). This drawback can be compensated for either by playback of animations, simple mass-spring systems or, if possible, extension of the physics engine. The manufacturers of physics engines are currently working on introducing new features like fluids and soft body simulation, so the features missing right now may be available in the near future (see chapter 6). 


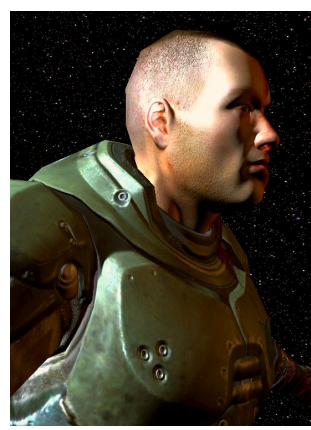

(a) Human skin

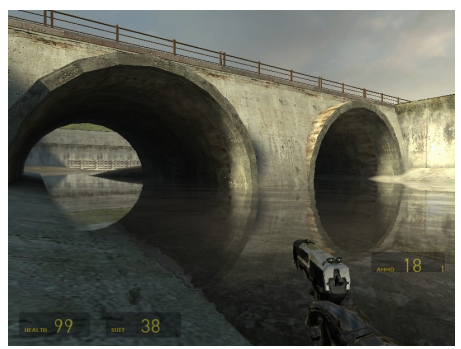

(c) Reflection

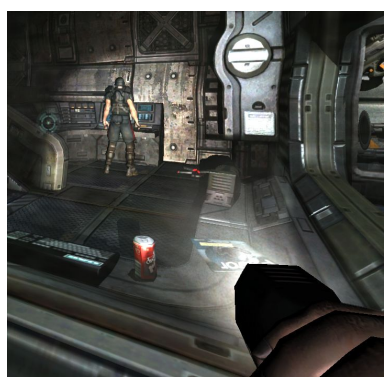

(e) Spotlights

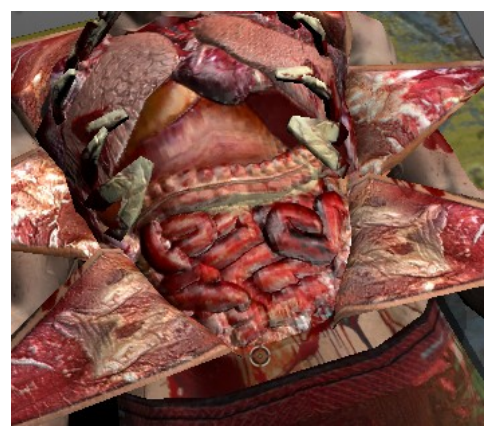

(b) Human tissue

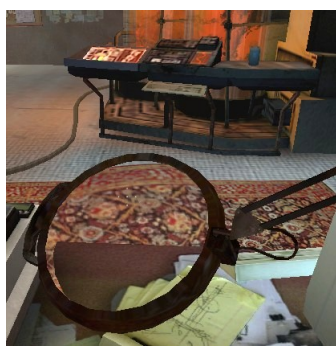

(d) Distortion

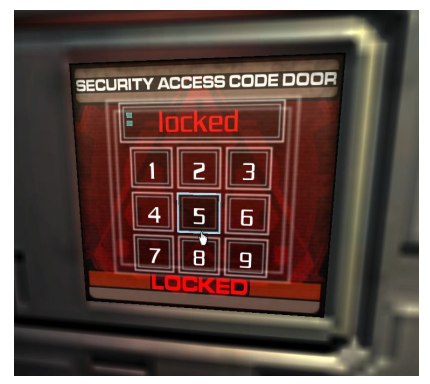

(f) User interfaces

Figure 4: Graphical capabilities of modern game engines. The screenshots are taken from the games Doom 3, Half-Life 2 and Quake 4.

As surgery is always a cooperative task, the networking (multiplayer) capabilities of games offer a great chance of building collaborative training scenarios. Players can see the position and state of their team members, and can communicate with each other by microphones and headphones or textual messages.

All of these features are useful for virtual surgery simulations. Advanced graphics makes the simulation more realistic and limited hardware requirements allow users in development countries and smaller clinics to employ the software. The network support and GUI customisation can bring together multiple users of surgical simulators for training of cooperative tasks, unrestricted by classroom walls and country boundaries and, when designed carefully, even independent of language barriers.

\section{Methodology}

\subsection{Engine Selection}

We started our selection of suitable game engines with an evaluation of an internet game engine database [DevMaster.net 2007]. At

the time of this evaluation (July 2007), this database contained 278 engines. We disregarded engines still in an early development state or those that were not developed or maintained any more. Engines without sound or other essential components were also removed from the list. Of the remaining engines, we selected those with inbuilt means of creating new game environments (maps). This last criterion is an important aspect for reducing the complexity of the editing process as there is no need for purchasing, installing and setting up external editors and necessary conversion tools, assumed the latter exist at all.

After the reduction of the original list by this selection process, we chose three inexpensive and in our opinion popular game engines for further evaluation.

- Unreal Engine 2 [Epic Games 2004]

- id Tech 4 [Wikipedia 2007]

- Source Engine [Valve Corporation 2004]

\subsection{Evaluation}

All engines were tested for their suitability for collaborative simulated surgical training applications by examining the following aspects:

Editing: Is everything that is necessary for creating and manipulating custom content included in the software? How is the editing process for a map started? Are the construction principles that are used during the process of building a map intuitive?

Content: How easy is the process of including game content as well as external models into the map? Which restrictions have to be considered when importing custom models?

Gameplay: How well can two or more users interact within the map and with the custom model? Are there any restrictions in the physical interaction?

Editing of models was performed with the 3D editor Blender [Blender Foundation 2007]. This software is free, in contrast to commercial and expensive 3D editors such as 3D Studio Max [Autodesk 2007a] or Maya [Autodesk 2007b], and has import and export filters for all important 3D formats that were necessary for including custom models into the maps created for each game engine.

\section{Results}

\subsection{Unreal Engine 2}

The following results are based on the game "Unreal Tournament 2004."

Editing The Unreal Engine 2 map editor "UnrealEd 3" is started as a standalone program. It incorporates model viewer, texture browser, script editor and other components necessary for editing a map (see figure 7(a)). In contrast to the editors of the other two engines, the editing process is of subtractive nature, i.e. volumes where players are allowed to move in have to be subtracted from the originally solid game world.

An editing concept common to all editors of the three evaluated game engines is the "brush." It is used for selecting e.g. the areas that will be subtracted from the game world. But it can also be used for adding walls, spheres, stairs or other simple geometries.

Geometrically complex objects like weapons are selected from a list, added into the map, and can then be placed, rotated, and 


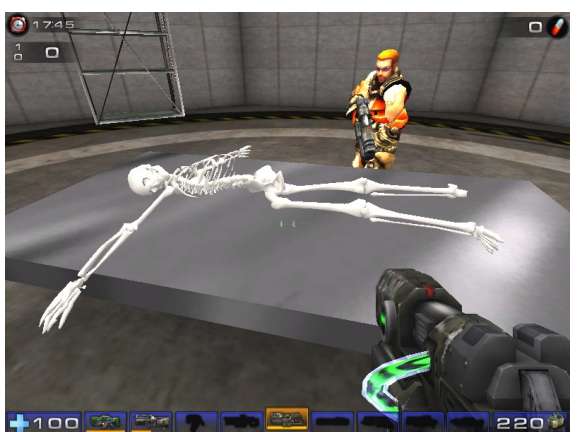

(a) Unreal Engine 2

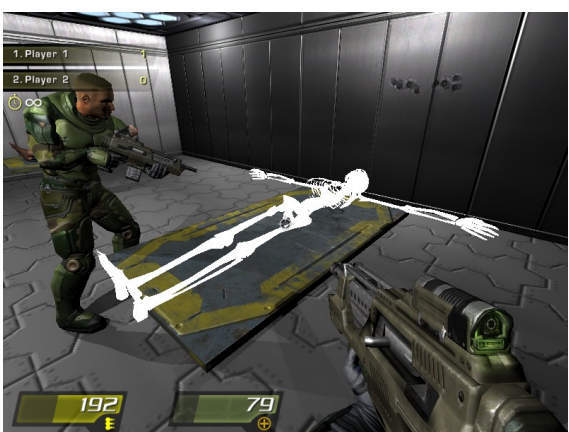

(b) id Tech 4

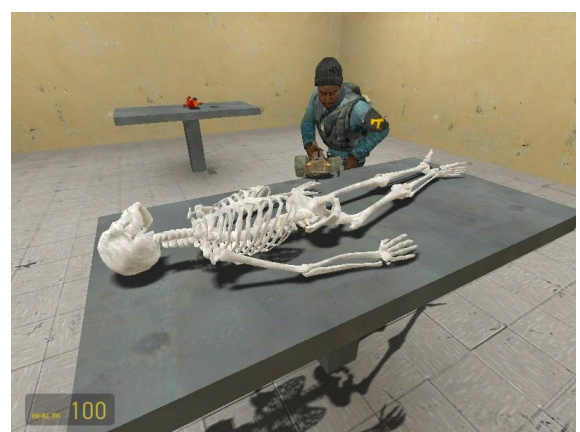

(c) Source Engine

Figure 5: Screenshots of our simulation scenarios implemented with different game engines.

changed in their behaviour or attributes. The same principle applies for physical objects like rigid bodies or joints.

Content We constructed a room with a metal shelf (game content) and a custom skeleton model on a table (custom content). The skeleton ${ }^{1}$ was split into parts (torso, skull, legs, and arms), which were then inserted as physical objects and connected by ball joints (see figure 6). The file format for inserting custom models can be one of . LWO (Lightwave Object File), or . ASE (ASCII Scene Exporter). We used the latter due to having an . ASE export filter in Blender,

Gameplay We started the map in multiplayer mode and interacted with the static and dynamic objects.
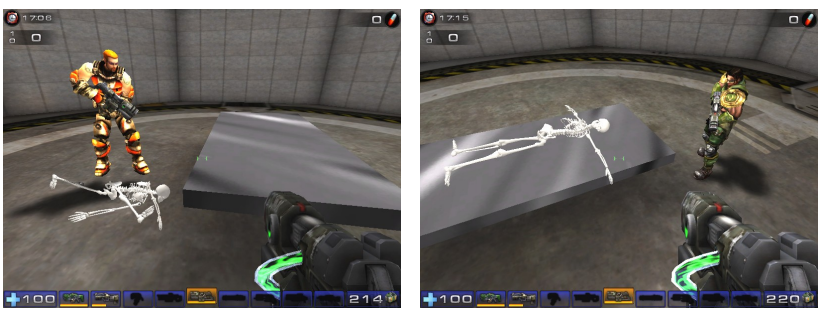

Figure 6: Asynchronous state of the skeleton on the server (left) and the client (right).

Non-physical actions and states are well synchronised between the server and the client. Player positions, orientations and states and also optical effects like decals (e.g. for bullet holes or scorchmarks) appear equally on both sides.

The articulated skeleton can be moved around by applying forces, e.g. with a weapon. This works well in single player mode and on the server side in multiplayer mode. However, the multiplayer client shows unexpected behaviour. When force is applied, the graphical representation of the skeleton stays in place, whereas its physical representation moves (see figure 6).

This asynchronism of the physics engine is not considered an error, as at 2003, the time of the release of the game, the physical simulation of game objects was not yet an important aspect of gameplay. Nevertheless, users wanted to create multiplayer maps with synchronised physical objects and thus developed a modification of the physics engine [Zepp 2005]. Due to the age of the Unreal

\footnotetext{
${ }^{1}$ Skeleton model source: http://artist-3d.com/free_3d_ models/dnm/model_disp.php?uid=637
}

Engine 2, this project has undergone no further improvement since 2005 and is now no longer available on servers.

\section{2 id Tech 4}

The following results are based on the game "Quake 4." This game uses a more recent version of the id Tech 4 engine than the game "Doom 3".

Editing The id Tech 4 engine incorporates a set of editors necessary for building maps and inserting custom content (see figure 7(b)). All of them can be started separately to edit, e.g., maps, articulated figures, effects, materials, and scripts.

The map editor "Radiant" has a simple user interface, including a world view and a texture and model browser. Like the other editors, it also uses the brush concept for adding simple geometries and a selection list for more complex objects. In contrast to the other two editors which use four windows for the top, front, side, and 3D view of the scene, this editor is restricted to a single window with the top view in conjunction with a simplified tall window for adjusting the height of placed objects.

Content With the map editor we created a simple room with two tables, on which we placed a game content model of a dissected body and a static custom content skeleton model, imported from an . ASE file (see figure 5(b)). We placed additional objects (fire extinguisher, book, gas bottle) in the scene to evaluate collaborative interactions with physical objects.

When we tried to articulate the skeleton by connecting the limbs and skull to the torso, we discovered that the physical support is limited to simple rigid bodies. This limitation was unexpected, due to the fact that we also worked with the id Tech 4 engine based game "Doom 3." In this game, a moveable crane with heavy, swinging load appears at least in one map. Its movements can be controlled by the user and the animation of the load is handled by the physics engine. Further investigation revealed that the physics of the game "Doom 3" is part of the game content, but not of the basic id Tech 4 engine [id Software 2007].

Gameplay The map was loaded in multiplayer mode and entered by two users. Player positions, orientations and states as well as optical effects are synchronised well between server and client.

Physical objects can be manipulated by both, although the refresh rate of the position and orientation of physical objects on 


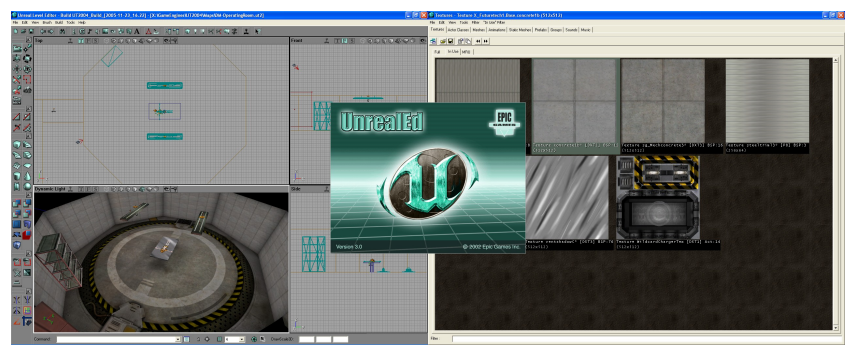

(a) "UnrealEd 3" (Unreal Engine 2)

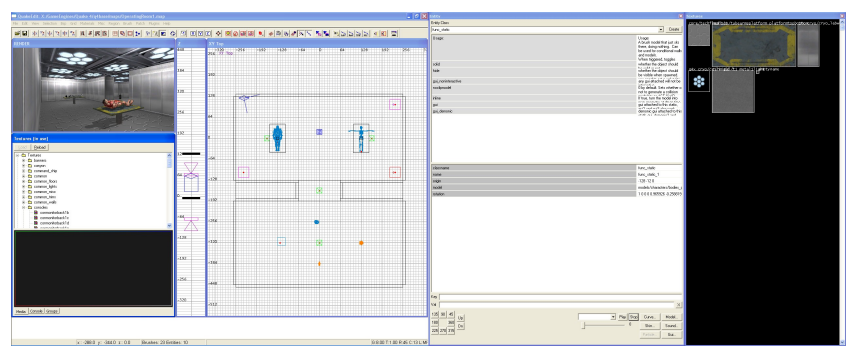

(b) "Radiant" (id Tech 4)

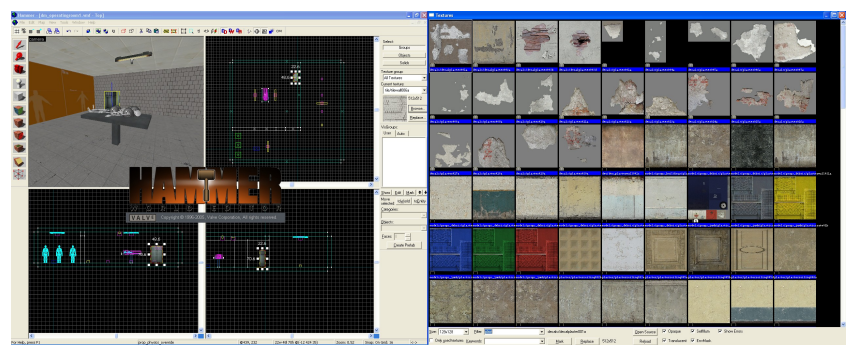

(c) "Hammer" (Source Engine)

Figure 7: Screenshots of the map editors of the three evaluated game engines.

the client is slow and results in a jerking movement. This problem could not be solved by manipulations of the server settings. Additionally, some physical items also showed the asynchronous behaviour of their optical and physical representations as for the Unreal Engine 2. It is yet unknown for which kind of objects this applies and if there are possible countermeasures.

\subsection{Source Engine}

The SDK of the Source Engine includes editors and helper programs and thus enables the construction of new maps and even modification of the source code of the engine. Permission to download it is obtained by purchasing a game of the "Half-Life 2" series.

Editing Maps are created and modified with the map editor "Hammer" (see figure 7(c)). The producer of the engine, Valve, also refers to the free XSI ModTool from Softimage [Avid Technology 2007], which, in conjunction with a special plugin that is available on the website of Valve, can be used for creating static and animated models.

The editor can be switched into different modes, like constructing solid objects, placing complex objects, moving objects, and texturing them. Complex objects (entities) are not only geometrically complex models, but also physical objects, physical constraints, light sources, pickable items (e.g. weapons, ammunition), etc.
An interesting and unique concept of the Source Engine is that of entity outputs and inputs. When, e.g., the output "OnTrigger" of a light switch entity is connected to the input "Toggle" of light source entity, the user can switch the light on and off by "using" the light switch with his character.

Content We created a test room with some game content objects (e.g. locker, lamps) and a table with the custom content skeleton. In contrast to the Unreal Engine 2 and the id Tech 4 engine, a model imported into the Source Engine may only consist of a maximum of 32768 vertices. This also limits the number of triangles to a maximum of about 11000 . These limits are coded into the engine and may not be changed. For performance reasons, Valve suggests to reduce the complexity of models to below 10000 triangles [Valve Developer Community 2007a]. We discovered that this limitation can be overcome by splitting the model into parts that are assembled into one object when converting the model data into the game engine's internal format, as described in the next paragraphs.

The whole process of creating custom models and textures is rather complex at first sight (see figure 8). For every model and texture, a "compiling instruction" file is necessary for converting it into the engine format (.qC and .txt). Especially the 3D model (.smd) is converted into a multitude of single files which hold information about geometry (.vvd), animations (.mol), physics (.phy) as well as special information for rendering the model in software, DirectX 8 and 9 (.sw.vtx, .dx80.vtx, $. d x 90 . v t x)$. The . vmt file gives additional information about the texture (e.g. specular lighting, normal mapping, physical surface properties) needed by the editor and the game engine.

The conversion programs and other tools are purely command line based. To convert a single model into the engine format, one has to drag the compilation file onto the converter in the explorer view, or start the process by entering a command line instruction. These command line based programs could be utilised easily to automatise a complex process of creating maps and models from medical datasets.

Gameplay Compared to the other two engines, the Source Engine performed best. The position, orientation and state of the users character as well as the physical simulation synchronised well and fluently on server and client (see figures 1 and 5(c)). The editor allows the selection of two kinds of physical objects: prop_physics and prop_physics_multiplayer. The latter reduces the amount of network traffic necessary for the synchronisation of physical objects on all connected machines, but also limits the interactivity of objects. They can only be moved by application of forces from weapons or tools, but not directly by users. This fact has to be considered when designing maps with objects that the user has to interact with as well as passive objects.

\section{Conclusion}

Modern game engines fulfill a great set of the features necessary for building a simulated surgical training application.

Graphics, audio and network capabilities are highly developed and allow the creator of applications to focus on content rather than details of the implementation. The underlying hardware is optimally used. Multiuser interaction is possible by multiplayer scenarios and allows the training of teamwork and cooperation.

Modern and highly mathematical physics models for simulation of soft tissue are (not yet) possible with game engines. Nevertheless, one can simulate basic soft tissue interaction by the use of simple mass-spring models. 


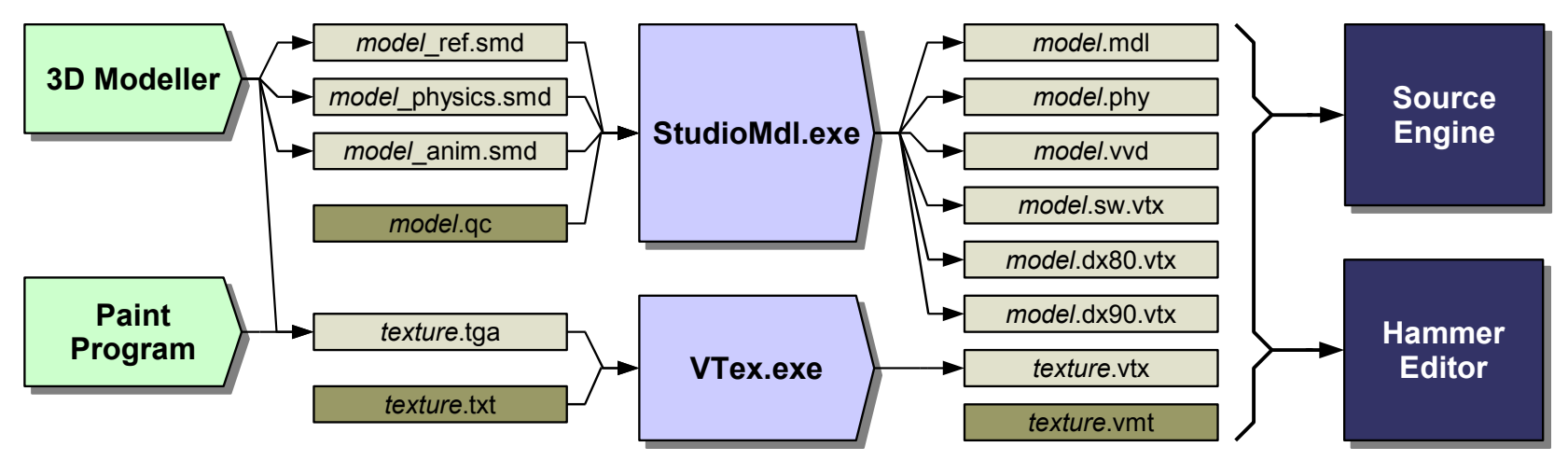

Figure 8: The flow of files and conversion for creating custom models and textures for the Source Engine.

Engines with publicly available source code like the Source Engine allow the extension of their physics engine features to mathematically more sophisticated models.

Predefined file formats can pose difficulties when converting medical images and models. These formats may be limited in their number of vertices or faces and thus would need preprocessing to reduce the amount of information without loss of optical detail. Another possibility for overcoming these limits is to split complex objects into parts that are kept together (e.g. by constraints [Wünsche et al. 2005]). On the positive side, the necessary file formats for the examined three engines are well documented (.ASE: [UnrealWiki 2007], . SMD: [Valve Developer Community 2007b]).

An interesting aspect of the Source Engine is the fact that not only the material and model compilation files are text based, but also the file format for maps. In conjunction with the command line based tools, this could lead to the development of a fully automatised tool that reads patient related medical data and constructs a map including the custom patient models for interaction and training.

\section{Future Work}

Automatisation The process of preparing and converting models for the use with game engines is up to date too complicated to be performed by untrained people. We plan to automatise this process by creating tools that can read and convert medical data directly into models and maps for use with game engines.

Feature Matrix We are currently preparing a matrix of features of surgical training devices for different kinds of medical procedures and features of game engines. With this matrix, the user can determine, which game engine would be best for implementing an application for a certain kind of procedure.

New Engines Two engines of the newest generation are expected to be released in the fourth quarter of 2007 :

- CryEngine 2 [Crytek 2002]

- Unreal Engine 3 [Epic Games 2006]

Among their features are enhanced graphics and physical modelling techniques. Animations can be blended while maintaining a set of constraints. Geometrical models can be deformed arbitrarily by displacement textures. Physically correct simulation of smoke and liquids is introduced. These features allow even more realistic surgical simulations and will be subject to further analysis.

\section{References}

ACGME. ACGME Outcome Project - General Competencies [online]. Sept. 1999 [cited 17.08.2007]. Available from: http: //www. acgme. org/out come/comp/compMin. asp.

Ageia Technologies. Ageia [online]. 2007 [cited 21.06.2007]. Available from: http://www.ageia.com/.

Allard, J., Cotin, S., Faure, F., Bensoussan, P.-J., Poyer, F., Duriez, C., Delingette, H., And Grisoni, L. 2007. SOFA - an Open Source Framework for Medical Simulation. In Medicine Meets Virtual Reality (MMVR 15).

AutOdEsK. Autodesk 3ds Max [online]. 2007 [cited 16.08.2007]. Available from: http://www. autodesk. com/3dsmax.

Autodesk. Autodesk Maya [online]. 2007 [cited 16.08.2007]. Available from: http://www. autodesk.com/maya.

Avid Technology. Welcome to Softimage - 3D Software Solutions for Games, Films, and Television Artists [online]. 2007 [cited 17.08.2007]. Available from: http://www. softimage.com/.

Bachofen, D., ZÁtonyi, J., Harders, M., SZÉkely, G., FrÜH, P., AND THALER, M. 2005. Enhancing the Visual Realism of Hysteroscopy Simulation. Studies in Health Technology and Informatics 119 (Jan.), 31-36.

BLENDER FOUNDATION. Blender [online]. 2007 [cited 17.08.2007]. Available from: http://www.blender. org/.

BRADLEY, P. 2006. The history of simulation in medical education and possible future directions. Medical Education 3, 3 (Mar.), 254-262.

ÇavuşoĞLu, M. C., GöKtekin, T. G., And Tendick, F. 2006. GiPSi: A Framework for Open Source/Open Architecture Software Development for Organ Level Surgical Simulation. IEEE Transactions on Information Technology in Biomedicine 10, 2 (Apr.), 312-322.

Chen, K.-T., Huang, P., And Lei, C.-L. 2006. Game traffic analysis: An MMORPG perspective. Computer Networks 50, 16 (Nov.), 3002-3023.

CISL. The MedSim-Eagle Patient Simulator [online]. 2007 [cited 17.08.2007]. Available from: http://med.stanford. edu/VAsimulator/medsim.html. 
Cotin, S., Delingette, H., And Ayache, N. 1999. RealTime Elastic Deformations of Soft Tissues for Surgery Simulation. IEEE Transactions on Visualization and Computer Graphics 5, 1 (Mar.), 62-73.

CRYTEK. CryEngine 2 Specifications [online]. 2002 [cited 17.08.2007]. Available from: http://www. crytek.com/ technology/index.php?sx=eng2.

Delingette, H. 1998. Toward Realistic Soft-Tissue Modeling in Medical Simulation. Proceedings of the IEEE 86, 3 (Mar.), $512-523$

DevMaster.net. 3D Game Engines Database [online]. 2007 [cited 17.08.2007]. Available from: http://www. devmaster.net/engines/.

DwOrZaK, J., AND Gu, L. 2007. Combining progressive and non-progressive cutting for soft tissue surgery simulations. International Journal of Computer Assisted Radiology and Surgery 2, Suppl 1 (June), S163-S165.

EPIC GAMES. Unreal Engine 2 [online]. 2004 [cited 17.08.2007]. Available from: http://www.unrealtechnology . com/html/technology/ue2.shtml.

EPIC GAMES. Unreal Engine 3 [online]. 2006 [cited 17.08.2007]. Available from: http://www.unrealtechnology. com/html/technology/ue30.shtml.

Geer, D. 2006. Vendors Upgrade Their Physics Processing to Improve Gaming. Computer 39, 8 (Aug.), 22-24.

HAVOK. Havok [online]. 2007 [cited 17.08.2007]. Available from: http: //www. havok.com/.

ID Software. id.sdk [The Code] [online]. 2007 [cited 17.08.2007]. Available from: http://www.iddevnet. com/doom3/code. php.

IMMERSION CORPORATION. Immersion Medical [online]. 2007 [cited 17.08.2007]. Available from: http://www. immersion.com/medical/.

LIM, Y.-J., AND DE, S. 2007. Real time simulation of nonlinear tissue response in virtual surgery using the point collocationbased method of finite spheres. Computer Methods in Applied Mechanics and Engineering 196, 31-32 (June), 3011-3024.

Mackenzie, J., Baily, G., Nitsche, M., And Rashbass, J., 2003. Gaming Technologies for Anatomy Education. Online, May. Available from: http: / /www.virtools.com/ news/pdf / 2004 /CARET .pdf [cited 17.08.2007].

Mentice. Mentice [online]. 2007 [cited 17.08.2007]. Available from: http://www. mentice.com/.

Montgomery, K., Bruyns, C., Brown, J., Sorkin, S., Mazzella, F., Thonier, G., Tellier, A., Lerman, B., AND Menon, A. 2002. Spring: A General Framework for Collaborative, Real-time Surgical Simulation. Studies in Health Technology and Informatics 85, 296-303.

Rodriguez-Florido, M. A., SÁnchez Escobar, N., SANTANA, R., And Ruiz-Alzola, J. 2006. An Open Source Framework for Surgical Simulation. Insight Journal (July). Available from: http://hdl.handle.net/1926/219 [cited 17.08.2007].

SELECT IT VEST Systems AG. Select-IT VEST Systems AG - medical science at your fingertips [online]. 2007 [cited 17.08.2007]. Available from: http://www.select-it. de/.
Serious Games Initiative. Games For Health [online]. 2007 [cited 17.08.2007]. Available from: http://www. gamesforhealth. org.

Serious Games Initiative. Serious Games Initiative [online]. 2007 [cited 17.08.2007]. Available from: http: / / www. seriousgames.org.

Simbionix. Simbionix, medical training simulators and clinical devices for MIS (minimally invasive surgery) [online]. 2007 [cited 17.08.2007]. Available from: http://www. simbionix.com/.

SIMSURGERY. Surgical training and Surgery education with SimSurgery [online]. 2007 [cited 17.08.2007]. Available from: http://www.simsurgery.no/.

Surgical ScIENCE. Surgical Science - Safer surgeons faster [online]. 2007 [cited 17.08.2007]. Available from: http: //www.surgical-science.com/.

UNREALWIKI. UnrealWiki: ASE File Format [online]. 2007 [cited 17.08.2007]. Available from: http://www. unrealwiki. com/wiki/ASE_File_Format.

VAlve CORPORATION. Valve Source Engine Features [online]. 2004 [cited 17.08.2007]. Available from: http: //www.valvesoftware.com/sourcelicense/ enginefeatures.htm.

Valve Developer Community. Compiling Models [online]. 2007 [cited 17.08.2007]. Available from: http: //developer.valvesoftware.com/wiki/ Compiling_Models_Basics.

VAlve DeVeloper Community. SMD file format [online]. 2007 [cited 17.08.2007]. Available from: http://developer.valvesoftware.com/wiki/ SMD_file_format.

VeREFI TECHNOLOGIES. Verefi Technologies, Inc. [online]. 2007 [cited 17.08.2007]. Available from: http://www.verefi. $\mathrm{com} /$.

Westwood, J. D., Haluck, R. S., Hoffman, H. M., MoGel, G. T., Phillips, R., RobB, R. A., And Vosburgh, K. G. 2005. Highly-Realistic, Immersive Training Environment for Hysteroscopy. Studies in Health Technology and Informatics 119 (Jan.), 176-181.

WiKIPEDIA. id Tech 4 - Wikipedia, The Free Encyclopedia [online]. 2007 [cited 17.08.2007]. Available from: http: //en.wikipedia.org/wiki/Doom_3_engine.

Wünsche, B. C., Kot, B., Gits, A., Amor, R., HoskING, J., AND GRUNDY, J. 2005. A Framework for Game Engine Based Visualisations. In Proceedings of Image and Vision Computing New Zealand 2005. Available from: http://www.cs.auckland.ac.nz/ burkhard/ Publications/IVCNZ05_WuenscheKotEtAl.pdf.

ZEPP, J. GoodKarma Physics Mod Beta 4 [online]. 2005 [cited 16.08.2007]. Available from: http: / / www ataricommunity.com/forums / showthread.php?t=440477. 


\section{Evaluation of Game Engines for Simulated Surgical Training}

Stefan Marks, John Windsor, Burkhard Wünsche

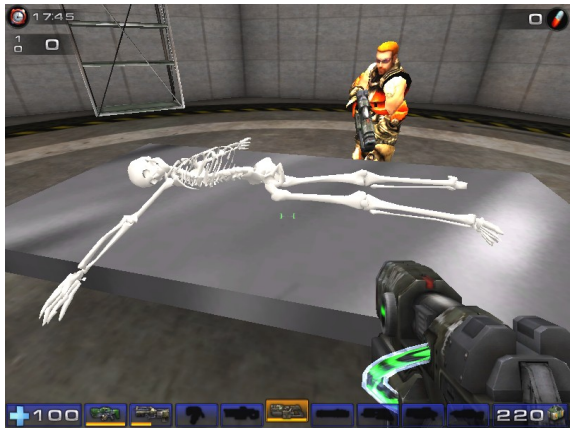

(a) Unreal Engine 2

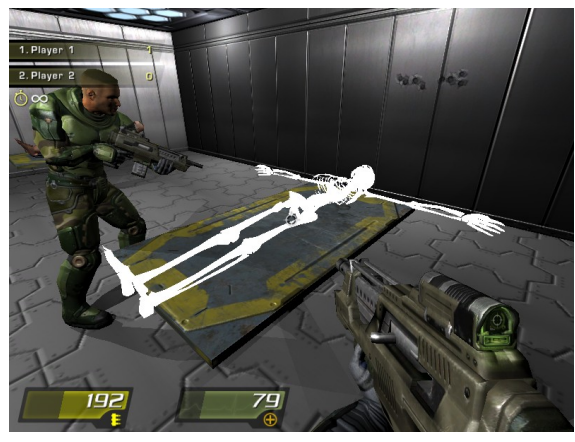

(b) id Tech 4

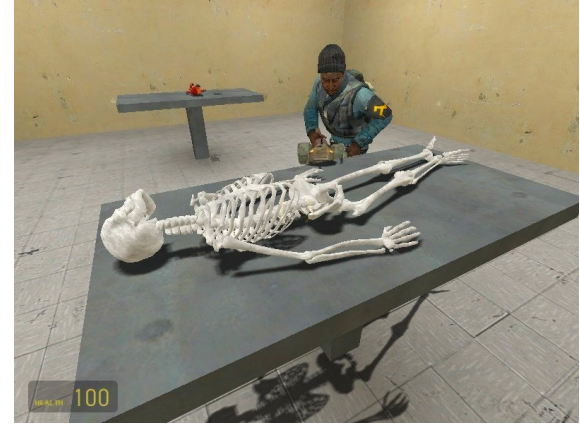

(c) Source Engine

Figure 1: Screenshots of our simulation scenarios implemented with different game engines.
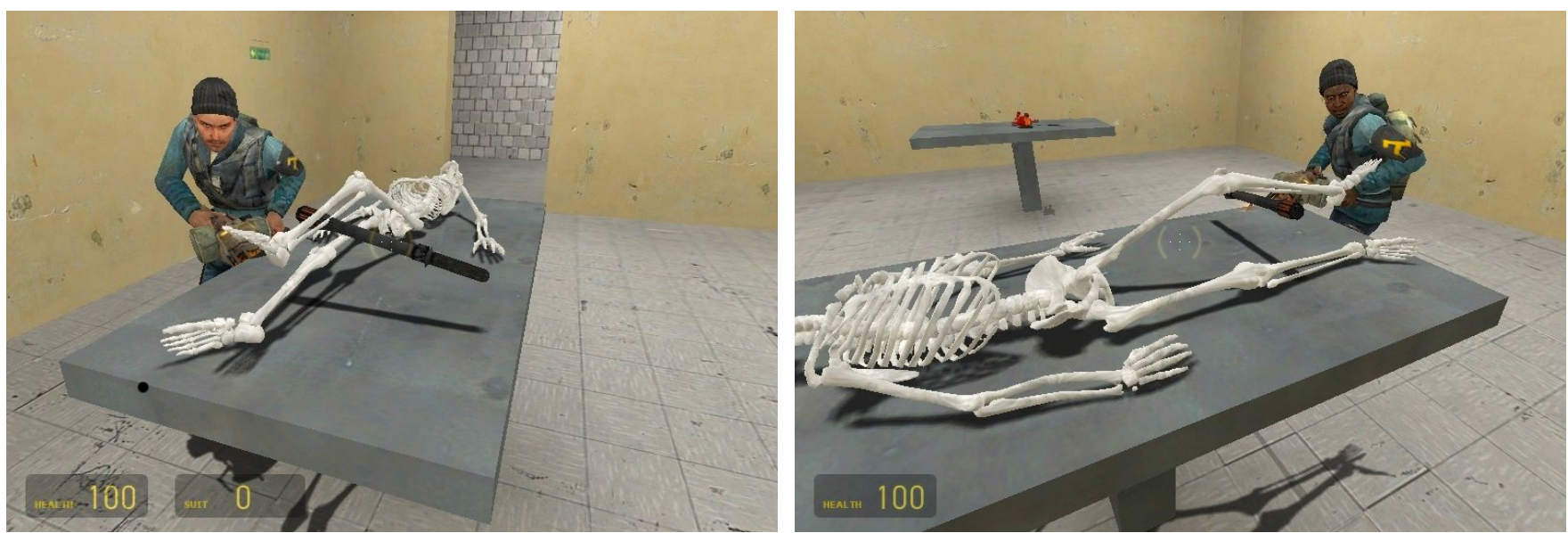

Figure 2: Cooperative interaction on a skeleton model. One user (left figure) is moving the leg with a stick. 